\title{
Variable criterion analysis of individual differences and stimulus similarity in choice reaction time
}

\author{
G. ROBERT GRICE, V. ALAN SPIKER, and ROBERT NULLMEYER \\ University of New Mexico, Albuquerque, New Mexico 87131
}

\begin{abstract}
The theory presented by Grice, Nullmeyer, and Spiker (1977) has been applied successfully to experiments with three levels of auditory similarity in choice reaction time. A defect in the original statement of the relation between theoretical probabilities and response probability is corrected. Similarity affects both positive associative strength and associative inhibition. The short-latency process of associative inhibition is especially sensitive to increasing levels of similarity. Descriptions of the speed-accuracy tradeoff as it depends upon stimulus similarity are presented. The forms of stimulus generalization gradients are shown to depend upon response latency. Common sets of theoretical functions described both group and individual subject latency distributions. As before, three individual difference variables are identified as mean criterion level, amount of criterion variability, and inhibitory ability. Multiple regression analysis of these three variables and error rate reveals interesting and interpretable features of information processing dynamics. One of these features is that the amount of criterion variability may be more important in determining error rate than the mean level of the criterion. Also included are comments on a critique by Link (1979) and a discussion of the relation between this approach and more formal deductive models.
\end{abstract}

Early applications of variable criterion analysis led to the development of a theory of simple reaction time (SRT) (Grice, Hunt, Kushner, \& Morrow, 1974) and disjunctive reaction time (DRT) of the c-reaction type (Grice, Hunt, Kushner, \& Nullmeyer, 1976). More recently, we have presented an empirically derived theory of choice reaction time (CRT) (Grice, Nullmeyer, \& Spiker, 1977). The result is a structure of some generality in that, when the same stimuli are used, functions obtained in SRT and DRT experiments become components of the CRT theory. According to this view, the excitatory strength (E) leading to response evocation increases as some deterministic function of time following stimulus onset. Response evocation occurs when the strength of $E$ reaches a preset criterion value $(C)$ which is specific to the trial. Over trials, the criterion is a normally distributed random variable with mean $\bar{C}$ and standard deviation $\sigma$. The mean and variability of the criterion distribution are determined by task variables, instructions, set, attention, motivation, adaptation, and individual differences.

As a scientific approach, our theoretical research is, at present, somewhat unconventional, and also,

This research was supported by PHS Grant MH 16400 from the National Institute of Mental Health. Appreciation is expressed to John M. Schnizlein, who assisted with the computations and participated in discussions of the research. Requests for reprints should be sent to G. Robert Grice, Department of Psychology, University of New Mexico, Albuquerque, New Mexico 87131. we fear, not fully understood. This is exemplified in a recent critique by Link (1979). While we believe his criticism to be correct in one important respect to be discussed later, it appears that his more general recommendations are based on a complete misunderstanding of our scientific goals and strategy. Our approach is not that of a simple, purely deductive model of the kind now most common in mathematical psychology. In addition to the usual deductive aspect of theory, we strongly emphasize inductive processes and techniques of data analysis based on the very limited model described in the opening paragraph. The goal of this latter aspect of work is the discovery of the existence and quantitative nature of the theoretical processes underlying a particular form of behavior. Processes discovered in this way then become part of a more complete theory which should provide a parsimonious and interpretable description of data, explicate the similarities and differences between different experimental situations, and have the properties of the more commonly understood deductive theory. We believe that such an explicit approach to theory development is, unfortunately, almost entirely neglected in contemporary theoretical research. One example is in the area of conditioning, where Grice $(1971,1972,1977)$ was able to isolate quantitatively the effects of learning, motivation, and sensory strength as reflected in both conditioning curves and distributions of conditioned response latency.

It is in this connection that Link's (1979) mis- 
understanding becomes clear in his criticism of the article by Grice et al. (1977). While the example he used was clearly not the theory under consideration, his basic recommendation was that all parameters of the theory be simultaneously estimated by means of methods such as chi-square minimization or maximum likelihood. The suggestion not only missed the point, it was obviously impossible. This was not a case of a theory under test, but of a theory under construction. As was explicit in the article, the forms of the functions for the growth of $E$ were unknown at the outset. The identity and forms of the component functions were discovered in the analysis, and within this process the parameters were obtained. It is also important that the parameters of two of the component functions did not come from the CRT data at all, but from other experimentsSRT and DRT. We can think of no instance in which the simultaneous estimation of all parameters would ever constitute a proper test of such a theory. Only the much smaller number of parameters relevant to the particular nature of the test should be free to vary. For example, when Grice et al. (1977) successfully tested the applicability of the theory to individual subjects, only the three parameters associated with individual differences were estimated and all parameters of the theoretical functions were invariant. Similarly, when the theory, derived from a speeded experiment, was applied to another experiment with an accuracy emphasis, a satisfactory fit was obtained when only the two criterion parameters were estimated.

A Thurstonian model for the theoretical analysis of data was presented in some detail by Grice et al. (1977). In experiments in which there is only a single response, the expected value of $E$ at any time, $t$, following stimulus onset is given by the normal deviate of the cumulative proportion of trials on which a response has occurred by that time. This value is on a scale with $\bar{C}$ as the origin and $\sigma$ as the unit. When based upon the entire cumulative distribution, these estimates describe the growth of $E$ as a function of time. The relations between the criterion parameters of two conditions or two subjects are estimated by means of a linear response evocation characteristic (REC) relating the corresponding values of $E$ at successive intervals of time. When such a linear relation exists, it implies that the two conditions or subjects share a common function for the growth of $E$. The slope estimates $\sigma_{\mathrm{x}} / \sigma_{\mathrm{y}}$, and the intercept estimates $\left(\overline{\mathrm{C}}_{\mathrm{x}}-\overline{\mathrm{C}}_{\mathrm{y}}\right) / \mathrm{o}_{\mathrm{y}}$.

The CRT theory assumes that, in the two-choice situation, there are two functions for the growth of $E^{*}$ following stimulus onset-one function for the correct response and one for the incorrect response. The growth of $\mathrm{E}$ for correct responses consists of two components-sensory strength (V) and associative strength (A) to the positive stimulus. E for errors involves the same processes. However, as time passes, the effect of sensory strength is reduced by an increasing ability to inhibit errors. This is associative inhibition (I). Also, the amount of generalized associative strength $\left(A_{g}\right)$ for the incorrect response is less than $A$ for the correct response. Associative discrimination $\left(A_{D}\right)$ progressively increases the difference between $A$ and $A_{g}$ as time increases.

The theory further assumes that there is a separate criterion distribution for each response and that the distributions are independent.' When one of the values of $E$ reaches criterion, that response occurs and the trial is terminated. Thus, the theory may be described as a race model. While growth of the correct $\mathrm{E}$ is greater than that for the incorrect response, errors may occur because, on some trials, the criterion for the incorrect response may be sufficiently lower than the correct criterion that the incorrect $E$ reaches criterion first. In this situation, the scaling model does not apply directly to the cumulative distributions for correct responses and errors. For example, when errors are present, the cumulative proportions of correct responses systematically underestimate the theoretical probabilities that $\mathrm{E}$ is equal to or greater than the criterion as these probabilities depend purely on the growth of $E$ and the criterion distribution. When an error occurs, that trial is eliminated from the total set as an opportunity for the correct response to occur and no information is provided as to what later time it would have reached criterion. In this paper, we present a correction of our original proposal (Grice et al., 1977) for estimating the theoretical probabilities from response probabilities and for calculating predicted response probabilities from theoretical probabilities. We reasoned, correctly, that the occurrence of an error eliminated that trial as an opportunity for the correct response to reach criterion later. However, we concluded, incorrectly, that the theoretical probability of the correct response occurring at a particular time was equal to the conditional probability of a response at that time, given that an error had not previously occurred. This resulted in a small underestimation of the theoretical probabilities, and is the basis upon which Link (1979) has correctly criticized our procedure. Special problems exist for us in this connection. Not only must we be able to calculate predicted response probabilities from theoretical functions for growth of excitatory strength, but we must also be able to determine the form of the underlying excitatory strength functions from empirical data. These functions are the inverse normal transformations of the theoretical probabilities of reaching criterion, and these probabilities must be determined first. We must begin with only the empirical response proportions. Now, the effects of the race are cumulative 


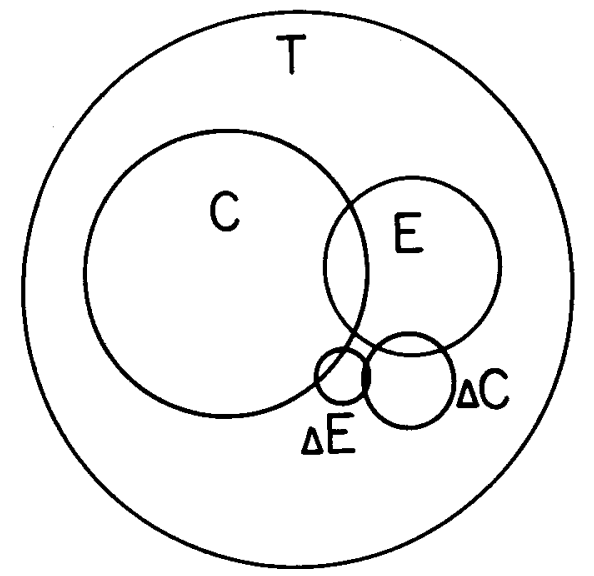

Figure 1. Venn diagram illustrating the race model. T represents the set of all trials described at time, $t$, following stimulus onset. Other terms represent subsets of $T$. $C$ is the theoretical subset on which the correct response has reached criterion by $t$. $E$ is the corresponding subset for the incorrect response. $\Delta C$ is the theoretical subset on which the correct response will reach criterion in a brief interval, $\Delta t$, beginning at $t . \Delta E$ is the corresponding subset for errors. The subset on which a response has occurred by $t$ is $C \cup E$.

and continuously changing as time elapses. It turns out that in order to estimate from data the probability of each response reaching criterion at time $t$, we must also know the theoretical probability that each response has reached criterion before $t$. These values may be obtained, however, if the computations are made successively for brief, finite intervals, and successively summed, beginning with the earliest interval with nonzero response frequency. Before that, the theoretical probability is zero. In doing this, we follow the conventional mathematical practice of assuming a smooth function to be linear within a sufficiently brief interval. Such a practice leads to negligible error.

With reference to Figure 1, let $T$ be the set of all trials to be described at time, $t$, following stimulus onset. $T$ is a sample from a population of such trials. $C$ is the subset of $T$ in which the excitatory strength of the correct response has reached criterion by $t$, including those trials on which it would have reached criterion had the trial not been interrupted by an error. $E$ is the corresponding subset in which the strength of the incorrect response has reached criterion or would have done so in the absence of a correct response. $\Delta C$ is the set of trials on which the correct response, as the results of the growth of excitatory strength, will reach criterion in the brief interval $\Delta \mathrm{t}$ beginning at $\mathrm{t} . \Delta \mathrm{E}$ is the corresponding increment for errors.

The subset of $\mathrm{T}$ on which one or the other of the responses has occurred is $\mathrm{C} \cup \mathrm{E}$, i.e.,

$$
R_{c}+R_{e}=C \cup E
$$

or

$$
\mathbf{R}_{c}+\mathbf{R}_{e}=\mathrm{C}+\mathrm{E}-\mathrm{C} \cap \mathrm{E},
$$

where $R_{c}$ and $R_{e}$ represent correct responses and errors. Now, the proportions of any set of trials on which either response is expected to reach criterion are the theoretical probabilities of reaching criterion. Thus, in terms of Figure 1, the expected relationships are $F_{c}(t)=C / T$ and $F_{e}(t)=E / T$, where $F_{c}(t)$ and $\mathrm{F}_{\mathrm{e}}(\mathrm{t})$ are theoretical probabilities of reaching criterion before $t$. Also, $\Delta F_{c}(t)$ and $\Delta F_{e}(t)$ are the theoretical probabilities of each response reaching criterion within $\Delta t$. Expressed as proportions of $T$, the predicted cumulative response probabilities, $P_{c}(t)$ and $P_{e}(t)$, are $P_{c}(t)=R_{c} / T$ and $P_{e}(t)=R_{e} / T$. Under independence, the probability of the joint occurrence of $\mathrm{C}$ and $\mathrm{E}, \mathrm{C} \cap \mathrm{E}$, is $\mathrm{F}_{\mathrm{c}}(t) \mathrm{F}_{\mathrm{e}}(t)$. Rewriting Equation 1 in terms of the probabilities gives the basic expression relating response proportions to the theoretical probabilities:

$$
P_{c}(t)+P_{e}(t)=F_{c}(t)+F_{e}(t)-F_{c}(t) F_{e}(t) \text {. }
$$

Since the relation of Equation 2 must hold after the increments in $\Delta t$, the following relation also holds:

$$
\begin{aligned}
P_{c}(t)+ & \Delta P_{c}(t)+P_{e}(t)+\Delta P_{e}(t) \\
= & F_{c}(t)+\Delta F_{c}(t)+F_{e}(t)+\Delta F_{e}(t) \\
& \quad-\left[F_{c}(t)+\Delta F_{c}(t)\right]\left[F_{e}(t)+\Delta F_{e}(t)\right]
\end{aligned}
$$

where $\Delta P_{c}(t)$ and $\Delta P_{e}(t)$ are the probabilities of correct responses and errors in $\Delta t$. Expanding Equation 3 yields

$$
\begin{aligned}
P_{c}(t)+\Delta P_{c}(t)+ & P_{e}(t)+\Delta P_{e}(t) \\
= & F_{c}(t)+\Delta F_{c}(t)+F_{e}(t)+\Delta F_{e}(t) \\
& -F_{c}(t) F_{e}(t)-\Delta F_{c}(t) F_{e}(t) \\
& -\Delta F_{e}(t) F_{c}(t)-\Delta F_{c}(t) \Delta F_{e}(t) .
\end{aligned}
$$

Subtracting the equality of Equation 2 leaves only the incremental terms as follows:

$$
\begin{aligned}
\Delta \mathrm{P}_{\mathrm{c}}(\mathrm{t})+\Delta \mathrm{P}_{\mathrm{e}}(\mathrm{t}) & \\
= & \Delta \mathrm{F}_{\mathrm{c}}(\mathrm{t})+\Delta \mathrm{F}_{\mathrm{e}}(\mathrm{t})-\Delta \mathrm{F}_{\mathrm{c}}(\mathrm{t}) \mathrm{F}_{\mathrm{e}}(\mathrm{t}) \\
& -\Delta \mathrm{F}_{\mathrm{e}}(\mathrm{t}) \mathrm{F}_{\mathrm{c}}(\mathrm{t})-\Delta \mathrm{F}_{\mathrm{c}}(\mathrm{t}) \Delta \mathrm{F}_{\mathrm{e}}(\mathrm{t}) .
\end{aligned}
$$

The final term in Equation 5 represents the probability indicated by the intersection of $\Delta \mathrm{C}$ and $\Delta \mathrm{E}$ in Figure 1. For a brief interval, this is a very small 
value and perhaps could be neglected. However, it is preferable not to do so, and an estimate of this term is introduced. Under the assumption that the growth of the theoretical probability of reaching criterion is linear within a brief interval, on those few trials when both responses reach criterion in the same interval, each will do so first about half of the time. To indicate the negligible nature of any error introduced by this assumption, the entire effect of this intersection term when cumulated over complete distributions is of the order of .01 , or less if the error rate is low. Under this condition, Equation 5 is separated into expressions for each response as follows:

$$
\Delta \mathrm{P}_{\mathrm{c}}(\mathrm{t})=\Delta \mathrm{F}_{\mathrm{c}}(\mathrm{t})-\Delta \mathrm{F}_{\mathrm{c}}(\mathrm{t}) \mathrm{F}_{\mathrm{e}}(\mathrm{t})-.5 \Delta \mathrm{F}_{\mathrm{c}}(\mathrm{t}) \Delta \mathrm{F}_{\mathrm{e}}(\mathrm{t})
$$

or

$$
\Delta \mathrm{P}_{\mathrm{c}}(\mathrm{t})=\Delta \mathrm{F}_{\mathrm{c}}(\mathrm{t})\left[1-\mathrm{F}_{\mathrm{e}}(\mathrm{t})\right]-.5 \Delta \mathrm{F}_{\mathrm{c}}(\mathrm{t}) \Delta \mathrm{F}_{\mathrm{e}}(\mathrm{t})
$$

and

$$
\Delta \mathrm{P}_{\mathrm{e}}(\mathrm{t})=\Delta \mathrm{F}_{\mathrm{e}}(\mathrm{t})-\Delta \mathrm{F}_{\mathrm{e}}(\mathrm{t}) \mathrm{F}_{\mathrm{c}}(\mathrm{t})-.5 \Delta \mathrm{F}_{\mathrm{c}}(\mathrm{t}) \Delta \mathrm{F}_{\mathrm{e}}(\mathrm{t})
$$

or

$$
\Delta \mathrm{P}_{\mathrm{e}}(\mathrm{t})=\Delta \mathrm{F}_{\mathrm{e}}(\mathrm{t})\left[1-\mathrm{F}_{\mathrm{c}}(\mathrm{t})\right]-.5 \Delta \mathrm{F}_{\mathrm{c}}(\mathrm{t}) \Delta \mathrm{F}_{\mathrm{e}}(\mathrm{t}) .
$$

Equations 6 and 7 are the means for calculating the predicted probabilities of correct responses and errors in $\Delta t$. Summation of these values over all $\Delta$ ts yields the predicted cumulative distributions of correct responses and errors. In prediction from functions for the growth of excitatory strength in scale units, the functions are first transformed to the functions for the growth of theoretical probability, $F_{c}$ and $F_{e}$, by the normal function. Values of $\Delta F_{c}$ and $\Delta F_{e}$ are then obtained by successive subtraction for the desired brief interval, $\Delta t$.

The relation of this analysis to Link's (1979) comment may now readily be seen. If $\Delta t$ is allowed to approach zero, the incremental terms in Equation 6 become the size of differentials and the intersection term vanishes. If the resulting expression is integrated over time, the following equation results:

$$
P_{c}=\int_{0}^{\infty} f_{c}(t)\left[1-F_{e}(t)\right] d t,
$$

where $P_{c}$ is the predicted cumulative proportion of correct responses and $f_{c}(t)$ is the ordinate of the noncumulative correct theoretical distribution at t. Equation 8 is identical to Link's Equation 1, upon which he bases his argument. A corresponding equation may be written for errors. While approached differently, the model is that which Link recommended. However, our derivation has been done in a way to include the terms necessary for accurate computation over finite intervals in order to meet our requirement. This possibility was not discussed by Link.
In order to obtain expressions for the extraction of the theoretical functions from data, we must also estimate the theoretical terms $\Delta F_{c}(t)$ and $\Delta F_{e}(t)$ from empirical response proportions. Rearranging Equation 6 , we obtain

$$
\Delta \mathrm{F}_{\mathrm{c}}(\mathrm{t})=\frac{\Delta \mathrm{P}_{\mathrm{c}}(\mathrm{t})}{1-\mathrm{F}_{\mathrm{e}}(\mathrm{t})}+\frac{.5 \Delta \mathrm{F}_{\mathrm{c}}(\mathrm{t}) \Delta \mathrm{F}_{\mathrm{e}}(\mathrm{t})}{1-\mathrm{F}_{\mathrm{e}}(\mathrm{t})} .
$$

Since the theoretical terms of the intersection term are not available, a very close approximation of the small correction term is used. The approximation of Equation 9 is

$$
\begin{aligned}
\Delta F_{c}(t)= & \frac{\Delta P_{c}(t)}{1-F_{e}(t)} \\
& +\frac{.5\left[\Delta P_{c}(t) / 1-F_{e}(t)\right]\left[\Delta P_{e}(t) / 1-F_{c}(t)\right]}{1-F_{e}(t)},
\end{aligned}
$$

where $\Delta \mathrm{P}_{\mathrm{c}}(\mathrm{t})$ and $\Delta \mathrm{P}_{\mathrm{e}}(\mathrm{t})$ are now the empirical response proportions in $\Delta t$. The corresponding equation for estimating $\Delta \mathrm{F}_{\mathrm{e}}(\mathrm{t})$ is

$$
\begin{aligned}
\Delta F_{e}(t)= & \frac{\Delta P_{e}(t)}{1-F_{c}(t)} \\
& +\frac{.5\left[\Delta P_{c}(t) / 1-F_{e}(t)\right]\left[\Delta P_{e}(t) / 1-F_{c}(t)\right]}{1-F_{c}(t)} .
\end{aligned}
$$

Equations 10 and 11 are computed sequentially over time, beginning with the earliest nonzero interval. Summations of the theoretical values $F_{c}(t)$ and $F_{e}(t)$ are made after each interval so that their values are available for the next calculation. The entire summations give the cumulative theoretical probabilities of each response reaching criterion as functions of time. The normal deviates of these probabilities, according to the scaling model, give functions for the growth of $E_{c}$ and $E_{e}$ in scale units. There should be no concern about the approximation in Equations 10 and 11. Equations 10 and 11 may be applied to a set of data and then Equations 6 and 7 applied to the resulting theoretical values. When this is done, data are returned with a cumulative error over all $\Delta$ ts no larger than an acceptable rounding error in the third decimal place. The accuracy of the calculations in both directions has been confirmed with simulated data. ${ }^{2}$

For clarification, a word should be added about the nature of these theoretical and predicted distributions. From Equation 2, it may be inferred that for the sum of the correct responses and errors asymptotically to approach unity, at least one of the 
theoretical cumulative functions must also approach one. For typical data, it is only the correct theoretical function which does so. In ordinary data, the probability of an error reaching criterion never approaches one. The predicted cumulative distributions for correct responses and errors reach the predicted total for each. They are not distributions of unit area, although the total of the two should approach one.

We have now recalculated the analyses of the previous paper (Grice et al., 1977) using the present Equations 10 and 11 , and the differences are all so small that no changes in any of our conclusions, including the forms of the component functions, are required. All measures of goodness of fit and individual difference phenomena are essentially identical.

The CRT theory, which we presented, was based on an experiment using as stimuli tones of 1,000 and $1,300 \mathrm{~Hz}$. It was conducted under speeded conditions employing a deadline. The growth of $\mathbf{E}$ for correct responses was analyzed into two components, sensory strength (V) and associative strength (A), relating the stimulus to the correct response.

For about the first $280 \mathrm{msec}$, the analysis indicated that correct response evocation depended entirely upon sensory strength, which grew with negative acceleration and was described by an exponential growth function. The function was obtained from a comparable SRT experiment with the same stimulus intensity and was mapped into the CRT data by criterion parameters estimated from a highly linear REC relating $\mathrm{V}$ to the growth of $\mathrm{E}$ for CRT for the first $280 \mathrm{msec}$. At about $280 \mathrm{msec}$, a second process began further to increase $E$ and grew in an inflected fashion. We attributed this to associative strength and described its growth by a Gompertz growth function. The expected excitatory strength at any time following stimulus onset was given by

$$
E_{C t}=\left(V_{t}+A_{t}-\bar{C}\right) / o,
$$

where $\mathrm{E}_{\mathrm{Ct}}$ is the correct excitatory strength at time $\mathrm{t}$. If $\overline{\mathrm{C}}$ and $\sigma$ are taken as 0 and 1 , these terms disappear.

Also within the theory, the growth of $\mathrm{E}$ for errors consisted of three components- $\mathrm{V}$, associative inhibition (I), and generalized associative strength $\left(\mathrm{A}_{\mathrm{g}}\right)$. The presence of inhibition, as in the earlier DRT theory (Grice et al., 1976), was inferred from the early divergence of the correct and error $E$ functions at a time when the correct response was dependent entirely upon $\mathrm{V}$. We reasoned that if correct responding was entirely dependent upon detection information, the depression in error rate must be based upon an ability to inhibit errors. I began very early and its growth was described by an inflected Gompertz function. The source of the I function was a DRT experiment, where the evidence indicated that it appeared in its pure form throughout its extent. It was mapped into the CRT data by criterion parameters and a weighting constant, $\mathbf{W}_{\mathrm{I}}$. $\mathbf{A}_{\mathrm{g}}$ followed the same time course as $\mathbf{A}$, was also described by a Gompertz function, but grew to a lower level than A. The expected strength of $E$ for errors was

$$
E_{e_{t}}=\left(V_{t}-I_{t}+A_{g t}-\bar{C}\right) / o
$$

In this paper, we make a terminological, but not a substantive, change in the statement of Equation 13. Rather than referring to generalized associative strength, we use its complement, associative discrimination, $A_{D}$, i.e., $A_{D}=A-A_{g}$. This modification has an important implication in emphasizing that $A_{g}$ is an attenuated form of $A$, and therefore must follow the same time course. Thus, an important feature of the structure of the data necessary to conform to our interpretation is that the effects of the late process, associative strength, must be observable in both the correct and error data at precisely the same time. It turns out that $A_{D}$ may also be described by an inflected Gompertz function. In this language, Equation 13 becomes

$$
E_{e t}=\left(V_{t}+A_{t}-I_{t}-A_{D t}-\bar{C}\right) / \sigma
$$

or

$$
\mathrm{E}_{\mathrm{et}}=\mathrm{E}_{\mathrm{ct}}-\mathrm{I}_{\mathrm{t}}-\mathbf{A}_{\mathrm{Dt}} \text {, }
$$

when $\bar{C}=0$ and $o=1$. One possible way of conceptualizing this theory in terms of simple flow chart is presented in Figure 2. We have not attempted to include the time characteristics of the processes in this figure.

The theory was initially based on the group RT distributions (subjects by trials) of correct responses and errors and provided an extremely accurate description of the data. Estimation of the first four moments of the group criterion distribution indicated that it closely approximated normality and justified the Thurstonian solution. However, an alternative solution based upon a between-subjects scaling procedure produced essentially identical results. The theory also accurately predicted the data of a second experiment with accuracy instructions when only the two criterion parameters were estimated. The fit was slightly better when a third parameter, $W_{I}$, was also estimated. An accurate description of the dynamics of the speed-accuracy tradeoff was provided, both between experiments with different speed instructions and as latency varied over trials within an experiment. The theory also gave a good account of individual subject data and provided a theoretical account of the nature of individual differences. The individual difference variables were the values of 


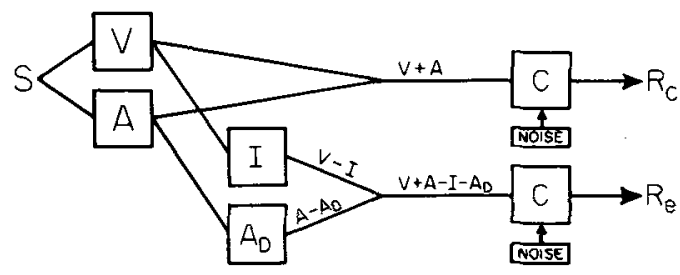

Figure 2. Diagramatic representation of the CRT theory. The presentation of a stimulus (S) results in two processes-sensory strength (V) and associative strength (A), which grow as different functions of time and combine additively. The criterion $(C)$ may be regarded as a gate with a specific sensitivity level. When the value of $\mathbf{V}+\mathbf{A}$ reaches this level, the correct response will occur. The value of $\mathrm{V}$ directed to the incorrect response is attenuated by associative inhibition, $I$, and the strength of $A$ by associative discrimination $\left(A_{D}\right)$. The two sources of attenuation have separate time characteristics. If the incorrect criterion is sufficiently low, the attenuated strength of $\mathbf{V}+\mathbf{A}$ may reach this level first and an error will occur. The noise inputs to the criteria symbolize the point that the sensitivity level is a random variable over trials. For simplicity, only one stimulus is represented. If the second stimulus were included, the left portion would be symmetrical and there would be two inputs to each gate.

$\overline{\mathrm{C}}$ and $\sigma$ for the criterion distribution and $\mathrm{W}_{\mathrm{I}}$ estimating inhibitory ability.

The purpose of this paper is to apply the theory to the analysis of stimulus similarity. Another purpose is to investigate, within this context, the applicability of the theory to both group and individual subject data and to analyze further the nature of individual differences.

\section{METHOD}

\section{Apparatus and Procedure}

Details of the apparatus and procedure have been previously described (Grice et al., 1977). The stimuli in the CRT experiments were tones of $100 \mathrm{~dB}$ SPL which differed in frequency. Subjects were instructed to respond with a right key to the higher tone and with a left key to the lower tone. In one experiment, the tones were 1,000 and $1,100 \mathrm{~Hz}$, and in the second they were 1,000 and $1,050 \mathrm{~Hz}$. Results from the previous experiment with 1,000 and $1,300 \mathrm{~Hz}$ are also included for comparison. Instructions were of the accuracy variety, i.e., to respond as fast as possible while avoiding errors. There were 24 practice trials followed without interruption by 120 presentations of each stimulus in an irregular order.

There were also two DRT experiments with the same stimuli. Only the right key was present and subjects were instructed to respond to the higher tone only. The number of stimulus presentations was the same as in CRT. Only portions of these data were used in the theoretical analysis of CRT.

\section{Subjects}

The subjects were 96 female students from a university course in introductory psychology who received course credit for participation. There were 24 in each experiment. However, in the two CRT experiments, 5 subjects were eliminated for failure to follow instructions. Thus, the analysis of the 1,100 experiment is based on 22 subjects, and that for the 1,050 experiment, on 21 subjects.

\section{RESULTS AND THEORETICAL ANALYSIS}

\section{Summary Statistics}

As summary statistics, we present the medians $(\mathrm{Mdn})$ and interquartile ranges $(\mathrm{Q})$ of the group distributions for CRT data. For correct responses in the 1,100 experiment, $\mathrm{Mdn}=416 \mathrm{msec}$ and $\mathrm{Q}=$ $129 \mathrm{msec}$. For errors, $\mathrm{Mdn}=385$ and $\mathrm{Q}=137$. For correct responses in the 1,050 experiment, Mdn $=404$ and $\mathrm{Q}=133$. For errors, $\mathrm{Mdn}=360$ and $\mathrm{Q}=154$. As previously reported, for correct responses in the 1,300 accuracy experiment, $\mathrm{Mdn}=$ 383 and $Q=119$; and for errors, $M d n=344$ and $\mathrm{Q}=108$. In all experiments, the typical finding of faster errors than correct responses was obtained. The mean error rate for the 1,100 experiment was $.053, \mathrm{SD}=.034$. In the 1,050 experiment, the error rate was $.122, \mathrm{SD}=.078$. The previously reported error rate for the 1,300 accuracy experiment was $.035, \mathrm{SD}=.020$.

\section{Estimation of the Theoretical Functions}

It is our purpose here to estimate from the present data the parameters of the same theoretical functions which successfully described the data of the two $1,300-\mathrm{Hz}$ experiments. This provides not only a test of the generality of the theory, but also a theoretical analysis of the effects of variations in stimulus similarity. As similarity varies, but intensity remains the same, we should expect no change in the function for $V$ except for a linear transformation dependent upon new criterion parameters. We should expect the functions for the associative processes to retain their mathematical forms, but to require new parameters. Especially, we expect both of the discriminative processes, I and $A_{D}$, to display less growth with increasing levels of similarity.

In the previous study, the primary analysis was based on the group distribution, and it was then shown that a between-subjects scaling solution based on an individual differences model produced essentially the same result. Here the analysis is based on the between-subjects scaling approach. Major concerns of this investigation are to evaluate the applicability of the theory to individual subjects and to study individual differences. For these purposes, this is the most direct approach. We shall also obtain from this solution, predictions of the group distributions.

The first step of the analysis is to obtain from the data of each subject estimates of the cumulative theoretical probability that the value of $\mathbf{E}$, for each response, is equal to or greater than the criterion at all times following stimulus onset. For correct responses and errors, this is estimated by the summation over brief finite intervals of Equations 10 and 11. The scaling model applies to these proportions, 
and their normal deviates estimate the growth of $\mathrm{E}$ as a function of time since stimulus onset. Thus, these functions were determined for each subject separately and for both correct responses and errors. The calculations were based on summations for 20-msec intervals, beginning with the earliest intervals with nonzero response frequencies. For the $1,100-\mathrm{Hz}$ experiment, the theoretical analysis was limited to intervals under $780 \mathrm{msec}$ and to those under $800 \mathrm{msec}$ for the $1,050-\mathrm{Hz}$ experiment.

The next step of the analysis is to obtain joint estimates of the functions for the growth of $E$ based on the means of all subjects, when all of the individual functions are on a common scale. This requires a complete between-subjects scaling solution in which the criterion parameters, $\bar{C}$ and $\sigma$, are estimated for each subject. For the $1,100-\mathrm{Hz}$ experiment, the procedure was the same as that reported by Grice et al. (1977), and all subjects were scaled with respect to a single reference subject whose correct response distribution substantially overlapped those of all other subjects. First, the time intervals in which the cumulative theoretical probability was nearest to the values of $.05, .10, .25, .50$, $.75, .90$, and .95 were identified for this subject. Then, by means of RECs, the scale values at these intervals were related to those of each other subject at the same times. Computation was by unweighted least squares mutual regression (Grice et al., 1974). All relations were substantially linear. The slopes and intercepts of the 21 regression equations give the estimates of $\sigma$ and $\bar{C}$ on the scale of the reference subject. The regression equations also provided the linear transformation by means of which all scale values for each subject were transformed to the scale of the reference subject. The result was a Subjects by Time Intervals matrix, with time at 20 -msec intervals and all entries on the common scale. The matrix was not complete because of lack of full overlap of the subject distributions. Means of the scale values of all subjects contributing in each time interval provide the joint estimate for the growth of E. This is the mean within-subjects function.

At this stage, improved estimates of the subject $\bar{C}$ and $\sigma$ parameters were obtained by 22 RECs relating the subject scale values to the mean scale values. Treating the mean values as error-free, unidirectional regression was used. In these computations, the values of $\mathrm{r}^{2}$ varied from .980 to .999 , with a mean of .994 and median of .997 . As a final step, all subject parameters and all mean scale values of $E$ were transformed to a more convenient scale for later use. In this scale, the unit was based on the mean of the subject variances, $1=\sqrt{\Sigma \sigma^{2} / N}$, and the origin was at the general mean, $0=\Sigma \bar{C} / \mathrm{N}$. Mean scale values for the growth of $E$ for errors were obtained in the same manner, except that transformations were by the subject parameters from the correct response analysis. Whenever there was a zero proportion in the error matrix in a cell which was filled in the correct matrix, it was filled by the normal deviate of $1 / 2 \mathrm{~N} .{ }^{3}$ The resulting mean scale values for the growth of $E$ are the plotted points in the middle panel of Figure 3.

In the $1,050-\mathrm{Hz}$ experiment, there was no single subject whose correct response distribution adequately overlapped those of all other subjects. Therefore, 2 reference subjects were used, and 12 subjects were scaled with respect to a relatively slow subject and 7 with respect to a relatively fast one. The mean scale values for the faster group were then converted to the scale of the slower group. This was accomplished by means of an REC relating the two sets of scale values in a range in which all subjects overlapped, from 300 to $500 \mathrm{msec}$. This relation was very linear, with $r^{2}=.995$. From this stage, the solution was the same. RECs relating the individual subject $E$ functions to the final mean scale values were linear, with values of $\mathrm{r}^{2}$ ranging from .965 to .999 and with a mean of .992 and a median of .994 . The final mean scale values for correct responses and errors are the points in the bottom panel of Figure 3.

The final stages in the application of the theory involve the estimation of the parameters of the component functions which successfully describe the two earlier $1,300-\mathrm{Hz}$ experiments. The function for the growth of the sensory strength component (V), as previously reported, is from an SRT experiment with stimuli of the same intensity and is an exponential growth function:

$$
V=2.283-13.583 \mathrm{e}^{-.00758 t} .
$$

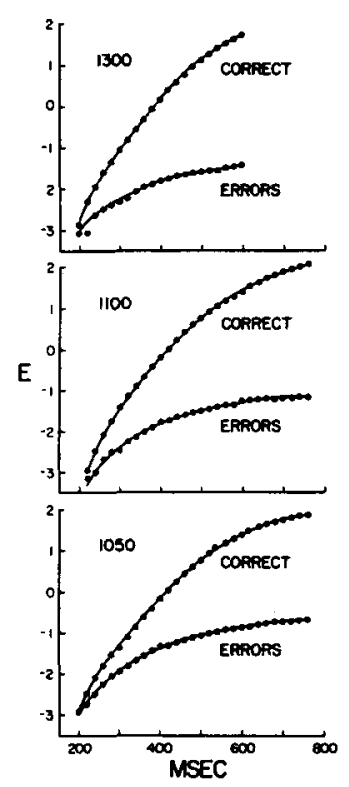

Figure 3. Functions indicating the growth of excitatory strength (E) for correct responses and errors for each of the three experiments. 
In the analysis of the $1,300-\mathrm{Hz}$ data, it was found that the early growth of correct response E for CRT was a linear function of $\mathrm{V}$ for intervals up to $280 \mathrm{msec}$. Beyond this point, E for CRT began to grow above the extrapolation of the linear relation, at first gradually, but with positive acceleration, and later was inflected to negative acceleration. This was interpreted to imply that the early responses were dependent only on sensory detection, and that the later growth represented the development of a second process-associative strength (A) relating the stimulus and the correct response. For the new experiments with increased similarity to confirm this interpretation, the same pattern must appear in the data. The slope and intercept of the early linear segment of the REC relating CRT to $V$ give $\sigma$ and $\bar{C}$ of the SRT experiment on the CRT scale, and provide the transformation by which V, Equation 16, may be mapped into the CRT data. The same situation was, in fact, true of the present data, except that the linearity lasted until about $300 \mathrm{msec}$, indicating a slightly later origin of the associative process. The least squares regression equations relating $E$ to $V$ for points below $300 \mathrm{msec}$ were for $1,100 \mathrm{~Hz}$ :

$$
\mathrm{E}_{\mathrm{CRT}}=1.292 \mathrm{~V}-2.572
$$

and for 1,050 Hz:

$$
\mathrm{E}_{\mathrm{CRT}}=.986 \mathrm{~V}-2.191 \text {. }
$$

Both functions were clearly linear in this region. For $1,100 \mathrm{~Hz}, \mathrm{r}^{2}=.999$; and for $1,050 \mathrm{~Hz}, \mathrm{r}^{2}=.998$. Equation 16 was then transformed to the scales of these experiments by Equations 17 and 18. The resulting two equations describe the growth of the sensory strength component in terms of the criterion parameters of these CRT experiments, i.e., on scales in which their respective values of $\overline{\mathrm{C}}$ and $\sigma$ are 0 and 1 .

From Equation 12, and when $\bar{C}$ and $\sigma$ are 0 and 1 , estimates of associative strength at any time are given by

$$
\hat{\mathbf{A}}_{\mathrm{t}}=\mathrm{E}_{\mathrm{ct}}-\mathrm{V}_{\mathrm{t}} \text {, }
$$

where $\hat{A}_{t}$ is the estimate of associative strength at any time, $E_{c t}$ is the mean scale value of correct excitatory strength at that time, and $V_{t}$ is computed from the transformation of Equation 16 at the same time. For each experiment, these estimates were obtained at 20 -msec intervals, beginning at $300 \mathrm{msec}$. In both cases, these estimates resulted in smooth inflected and increasing functions of time. As in the previous analysis, these functions are accurately described by Gompertz growth functions. The two equations are, for $1,100 \mathrm{~Hz}$ :

$$
\mathrm{A}=2.050\left(1.44 \times 10^{-15}\right)^{.9930^{1}}
$$

and for $1,050 \mathrm{~Hz}$ :

$$
A=1.980\left(2.186 \times 10^{-25}\right)^{.9910^{t}},
$$

where $\mathrm{A}$ is the amount of associative strength and $\mathrm{t}$ is the time in milliseconds since stimulus onset. Excitatory strength of the correct response at any time is the sum of the values calculated from the transformations of Equation 16 and from Equations 20 or 21 according to Equation 12 .

The first step in estimating the component functions for the growth of $E$ for errors is to obtain from the DRT experiments the functions describing the growth of associative inhibition (I). In the case of the $1,300-\mathrm{Hz}$ data, this was quite simple and straightforward. This was because the DRT data represented a pure case of the inhibitory strategy originally described by Grice et al. (1976). They found systematic differences between subjects which were interpreted as differences in the strategy with which a subject performed the DRT task. For some subjects, functions for the growth of $\mathrm{E}$ for correct responses were linear with the growth of $\mathrm{V}$ from SRT throughout the entire distributions, implying that responses to the imperative stimulus were on the same basis as in SRT, i.e., detection information. For these subjects, the occurrence of false alarms (FAs) decreased progressively with time. E for FAs was not linear with V. Since the evidence indicated that correct responses were on the basis of detection, it was inferred that these subjects could selectively inhibit responses to the catch stimulus with increasing effectiveness as time passed. This process was called associative inhibition (I), and its amount at any time was simply the difference between the $\mathrm{V}$ function and the $\mathrm{E}$ function for FAs. Other subjects showed only an early linear relation of the correct response function to $\mathrm{V}$, followed by a secondary rise similar to that found in CRT. This was termed the associative strategy. These subjects also showed evidence of the early inhibitory process. However, to obtain a pure function for the growth of I, applicable to CRT, only data from inhibitory subjects should be used because of the possible presence of $A_{g}$ in the FA functions of associative subjects. More recently, Spiker (1978) has confirmed the existence of the two kinds of subjects in DRT, but in addition, has found that the proportion of inhibitory subjects decreased with increasing difficulty of the task. It is not surprising, therefore, that in the present DRT experiments, conducted with the increased similarity as sources of the I functions, not all subjects were of the inhibitory variety. Thus, it was necessary to identify the inhibitory subjects and base the estimates of I on their data only.

For the pattern of the new CRT data to conform to the inhibitory interpretation, it is necessary that the early portion of the correct $E$ functions be linear 
with $\mathrm{V}$, and that the correct and error functions diverge during this period of linearity. It is further necessary that this divergence be described by an appropriate linear transformation of the I function from DRT. This was true of the two $1,300-\mathrm{Hz}$ experiments, and turns out to be true of both of the new experiments as well.

In DRT, with the inhibitory strategy, the expectations of $\mathrm{E}$ for correct responses and false alarms are given by

$$
E_{c t}=\left(V_{t}-\bar{C}\right) / \sigma
$$

and

$$
\mathrm{E}_{\text {FAt }}=\left(\mathrm{V}_{\mathrm{t}}-\mathrm{I}_{\mathrm{t}}-\overline{\mathrm{C}}\right) / \mathrm{o}
$$

Thus, when $\bar{C}$ and $\sigma$ are 0 and $1, I_{t}$ is estimated by

$$
\dot{\mathbf{I}}_{\mathrm{t}}=\mathrm{V}_{\mathrm{t}}-\mathrm{E}_{\mathrm{FAt}} \text {, }
$$

where the values of $E_{F A t}$ are the normal deviates of successive proportions from the cumulative distribution for FAs and calculated values of $\mathrm{V}$ are on the DRT scale. It will also be noted that the values of $V$ are also the calculated values of $E_{c t}$. For each DRT experiment, RECs were plotted relating the growth of $\mathrm{E}$ for each subject to the calculated $\mathrm{V}$ function, Equation 16. Our criterion for the selection of inhibitory subjects was that there must be a linear relation which was established before $300 \mathrm{msec}$ and which continued until at least $400 \mathrm{msec}$. There were 16 such subjects in the $1,100-\mathrm{Hz}$ experiment and 10 in the $1,050-\mathrm{Hz}$ experiment. Estimates of I were based on the group distributions of these subjects. Estimates of the third and fourth moments of the theoretical group criterion distributions (Grice et al., 1977) indicated that they closely approximated normality. The growth for correct responses of $E$ for each group was then related to $\mathrm{V}$ at 20 -msec intervals up to $400 \mathrm{msec}$. Both relations were very linear. For $1,100 \mathrm{~Hz}, \mathrm{r}^{2}=.998$, and for $1,050 \mathrm{~Hz}, \mathrm{r}^{2}=$ .999. By means of these regression equations, Equation 16 for $\mathrm{V}$ was then transformed to the scales of the two DRT experiments. I was then estimated by Equation 24 at $20-\mathrm{msec}$ intervals. The results, as in the previous analysis, were smooth, predominately negatively accelerated functions with slight initial positive acceleration. There were few errors above $400 \mathrm{msec}$, and there was no hint of discontinuity in the functions above this range. This means that they were uncontaminated by the presence of generalized positive associative strength, $A_{g}$. As before, they were fitted by Gompertz functions. The transformations for converting I to the CRT units is inferred from the slopes of the RECs relating both CRT and DRT to V. These factors are .939 for $1,100 \mathrm{~Hz}$ and
.737 for $1,050 \mathrm{~Hz}$. When multiplied by these values, the equations were on the CRT scales.

In the previous analysis, we found that the I functions from DRT did apply to CRT, but required a weighting factor $\left(\mathrm{W}_{\mathrm{I}}\right)$ less than one. These estimates are obtained by the slope of a line through the origin relating I from DRT to estimates of I for CRT before the origin of A, i.e., up to $300 \mathrm{msec}$. Since A is zero in this region, these estimates for CRT are also obtained by Equation 24. The estimates of $\mathrm{W}_{\mathrm{I}}$ obtained in this way were .673 for $1,100 \mathrm{~Hz}$ and .699 for $1,050 \mathrm{~Hz}$. When converted to the CRT scales and appropriately weighted, the Gompertz functions describing the growth of I for CRT are as follows:

$$
\text { for } 1,100 \mathrm{~Hz}: \mathrm{I}=1.909\left(9.621 \times 10^{-9}\right)^{.9891^{\mathrm{l}}}
$$

and

$$
\text { for } 1,050 \mathrm{~Hz}: \mathrm{I}=1.391\left(5.947 \times 10^{-10}\right)^{.9892^{1}} .
$$

The functions are the same as for DRT, except for the multiplicative constants, which are the asymptotes.

In obtaining the functions for the growth of associative discrimination $\left(A_{D}\right)$, estimates of generalized associative strength $\left(A_{g}\right)$ were obtained first. From Equation 13, when $\bar{C}$ and $\sigma$ are 0 and 1 , this estimate is

$$
\hat{A}_{\mathrm{gt}}=\mathrm{E}_{\mathrm{et}}-\mathrm{V}_{\mathrm{t}}+\mathrm{I}_{\mathrm{t}} \text {, }
$$

where $\hat{\mathrm{A}}_{\mathrm{gt}}$ is the estimate at time $t, \mathrm{E}_{\mathrm{et}}$ is the mean scale value for errors, and $V_{t}$ and $I_{t}$ are calculated from the theoretical equations. These estimates were obtained at 20 -msec intervals, beginning at $300 \mathrm{msec}$. Estimates of $A_{D}$ were then obtained by

$$
\hat{\mathbf{A}}_{\mathrm{Dt}}=\mathbf{A}_{\mathrm{t}}-\hat{\mathbf{A}}_{\mathrm{gt}} \text {, }
$$

where values of $A_{t}$ are calculated from Equations 20 and 21. The results were smooth functions of time which were accurately fitted by inflected Gompertz functions as follows:

$$
\text { for } 1,100 \mathrm{~Hz}: A_{D}=1.480\left(6.621 \times 10^{-41}\right)^{.9914^{t}}(29)
$$

and

$$
\text { for } 1,050 \mathrm{~Hz}: A_{D}=1.195\left(1.281 \times 10^{-71}\right)^{.9892^{t}},(30)
$$

with $t$ in milliseconds. It is now seen that the structure of the data in both experiments meets the final requirement of the theory. Excitatory strength for errors begins to rise above the value of $\mathrm{V}-\mathrm{I}$ at just the same time that the additional rise attributed to $\mathrm{A}$ occurs in the function for correct responses. The time 


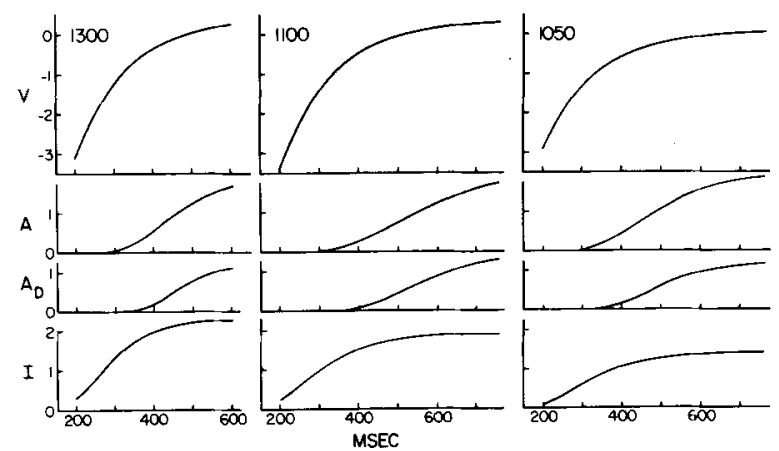

Figure 4. Functions for the components of excitatory strength for each of the experiments representing three degrees of stimulus similarity. The component functions indicate the temporal growth of sensory strength $(V)$, associative strength of the correct response (A), associative discrimination $\left(A_{D}\right)$, and associative inhibition (I).

course of this rise may be described accurately by subtracting the smooth function $A_{D}$ from $A$. This feature of the data clearly supports the assumption of late-developing associative strength which generalizes to the incorrect response with decreasing strength as time elapses.

Graphs of all component functions for the 1,100 and $1,050-\mathrm{Hz}$ experiments are presented in the center and right panels of Figure 4. Also included, for comparison purposes in the left panel, are the corresponding functions for the $1,300-\mathrm{Hz}$ accuracy experiment. These are from the recomputation of the previous analysis by Grice et al. (1977). The solution was modified to include the function for $A_{D}$ rather than $A_{g}$. The complete functions for the growth of excitatory strength are the smooth curves of Figure 3. These were obtained by combining the component functions according to Equations 12 and 15 . The only systematic discrepancy is at short latencies of the error function for $1,300 \mathrm{~Hz}$. However, the discrepancy is a region of unreliable scale values based on error proportions of .01 and under. It is negligible with respect to the prediction of response probabilities.

The growth of $E$ is composed of two processessensory and associative strength for correct responses. For errors, it is composed of the same two processes as attenuated by associative inhibition and associative discrimination. The biphasic character of the growth may be observed in the curves of Figure 3. However, it becomes clearer if one directly examines the slopes of the functions. For this purpose, we present, in Figure 5, the time derivatives of the six functions plotted as continuous functions of time.

\section{Prediction of the Group Distributions}

It is not conventional in RT research either to present or to attempt to interpret group RT distributions in which all subject distributions are combined. We believe that these distributions may be very useful. When properly interpreted, they provide a degree of generality not present in the distributions of individuals. Also, they provide very orderly data. The present theory appears to be unique in providing an account of individual differences which makes such distributions interpretable. The theoretical functions for the growth of E apply directly to the group CRT distributions if the group criterion distributions are approximately normal. This situation existed in the previous analysis of the two $1,300-\mathrm{Hz}$ experiments. In that paper (Grice et al., 1977), we presented expressions for the first four moments of the mixed criterion distribution, which may be calculated when estimates of $\bar{C}$ and $\sigma$ are available for each subject. The expressions for the third and fourth moments are estimates based on the assumption of normality within subjects. When these terms are computed for the present data, they result in the following estimates of skewness and kurtosis: for skewness, $\gamma_{1}=$ -.021 for $1,100 \mathrm{~Hz}$ and .058 for $1,050 \mathrm{~Hz}$; and for kurtosis $\gamma_{2}=.327$ for $1,100 \mathrm{~Hz}$ and .205 for $1,050 \mathrm{~Hz}$. Both $\gamma_{1}$ and $\gamma_{2}$ are zero for normal distributions. Thus, skewness is negligible and there is evidence for slight leptokurtosis. The two criterion distributions resemble t distributions of 22 and $33 \mathrm{df}$. Neither of these would introduce noticeable distortion when normal scaling procedures are used. It may be concluded from this that functions for the growth of $E$ derived from the group distributions should be the same as those from the between-subjects scaling solution except for a linear transformation relating the two scales.

The desired transformation has a rational basis and need not be derived from a linear regression

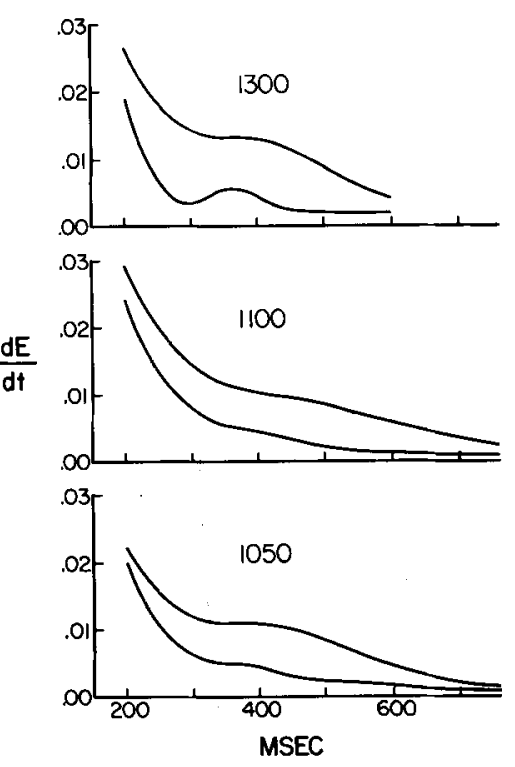

Figure 5. Time derivations of the functions for the growth of excitatory strength $(E)$ as presented in Figure 3. 
solution. Functions obtained from the group distribution have the general criterion mean as their origin and are in units of the standard deviation of the group distribution. The origins of the functions from the scaling solution were selected to be the same, so the transformation will have no additive constant. The variance of the group criterion distribution is

$$
\sigma_{\mathrm{G}}^{2}=\sigma_{\mathrm{s}}^{2}+\frac{\Sigma_{1}^{\mathrm{N}} \sigma_{\mathrm{k}}^{2}}{\mathrm{~N}},
$$

where $\sigma_{\mathrm{s}}^{2}$ is the variance of the subject criterion means and $o_{k}^{2}$ is the variance of subject $k$ 's criterion distribution. The unit of functions from the scaling solution was selected to be $\sqrt{\sum_{1}^{N} \sigma_{k}^{2} / N}$. It may therefore be concluded that the expression relating the two scales is

$$
\mathrm{E}_{\mathrm{Gt}}=\frac{1}{\sqrt{\sigma_{\mathrm{s}}^{2}+1}} \mathrm{E}_{\mathrm{BSt}},
$$

where $E_{G t}$ is a scale value derived from the group distributions at time $t, E_{B S t}$ is from the betweensubjects scaling solution, and $\sigma_{\mathrm{s}}^{2}$ is the variance of the subject criterion means. In evaluating this relation, scale values for correct responses and errors were calculated from the group distributions at 20 -msec intervals by Equations 10 and 11 . For correct responses, these were then plotted against the corresponding mean scale values, and the relations were linear. The scaling factors from Equation 32 were .952 for $1,100 \mathrm{~Hz}$ and .902 for $1,050 \mathrm{~Hz}$. The proportions of variance of the group scale values accounted for by the predicted relationships were .999 for $1,100 \mathrm{~Hz}$ and .998 for $1,050 \mathrm{~Hz}$. This is rather remarkable since this is a rationally predicted linear relation rather than a line of best fit.

The evidence indicates that the theoretical functions derived from the individual differences solution clearly do apply to the group distributions. This implies that the group distributions may be predicted from the theory. Thus, the theoretical functions of $E$, as presented in Figure 3, were converted to the group scales by Equation 32. Predicted distributions for correct responses and errors were then calculated by Equations 6 and 7. The calculated and obtained cumulative group distributions are presented in Figure 6. Distributions for the $1,300-\mathrm{Hz}$ accuracy experiment are presented there also. The proportions of variance accounted for correct responses are .999 for both 1,100 and $1,050 \mathrm{~Hz}$. For errors, the proportions of variance are .991 and .994 . The proportions for $1,300 \mathrm{~Hz}$ were .9997 for correct and .989 for errors. Of course, the relatively flat error distributions have less variance to account for. However, it is also true that the scaling factors and the criterion parameters

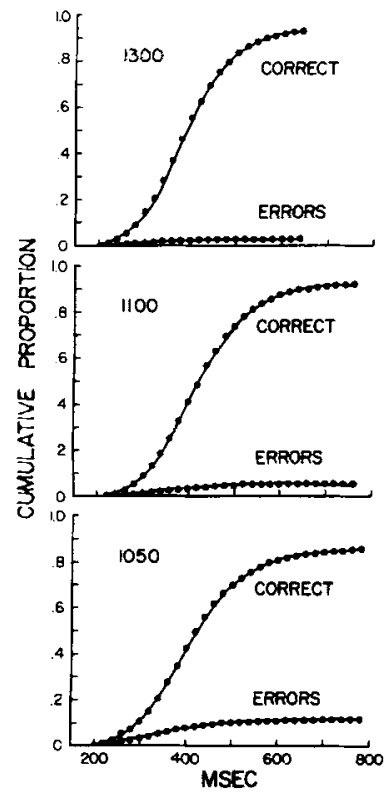

Figure 6. Calculated and obtained group, cumulative distributions for each of the three experiments.

were based on correct responses only. In the present context, it is significant that the theory does accurately describe the group distributions, since these data provide a satisfactory way of describing relations between stimulus similarity and latency phenomena.

\section{The Effects of Stimulus Similarity}

In theoretical terms, the latency effects of stimulus similarity may be observed in the functions for the growth of $E$ as presented in Figure 3 . The growth of $E$ for correct responses decreases with increasing levels of similarity, and the growth of $E$ for errors increases with similarity. However, it is the effect on the error functions which is most pronounced, and is primarily responsible for the differing rates at which the pairs of functions diverge. The divergence may be observed directly in Figure 7 where we have plotted, for each experiment, the difference in $E$ for correct responses and errors $\left(E_{c}-E_{e}\right)$. These functions at each point, are also equal to $I+A_{D}$, thus displaying the development of the total of the two discriminative processes. Roughly, they may be described as approximately linear segments followed by negative acceleration. Actually, all are curvilinear throughout their extents. Generally, discrimination grows monotonically with time, at rates inversely related to stimulus similarity. The asymptotic levels are also affected by similarity. These functions bear at least a superficial resemblance to the tradeoff functions ("latency operating characteristics") presented by Lappin and Disch (1972) in which a d' measure was plotted as a function of time. However, 


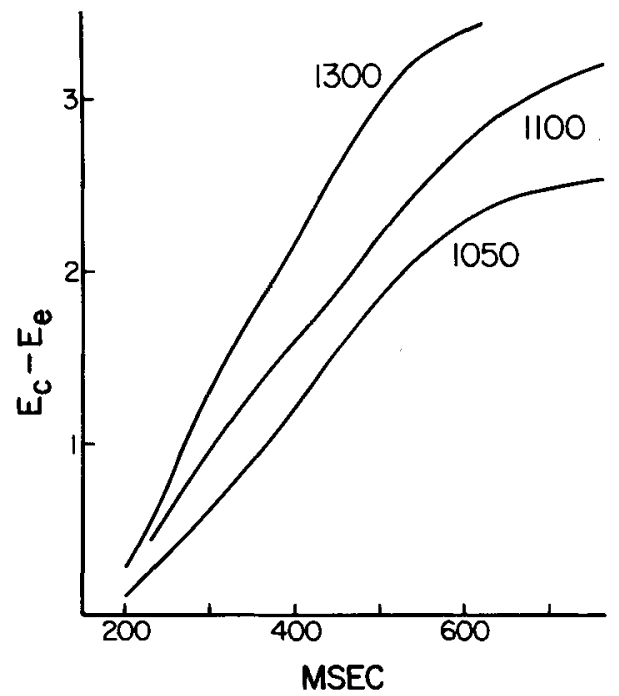

Figure 7. Theoretical speed-accuracy tradeoff functions expressed as the difference in excitatory strength between correct responses and errors $\left(E_{c}-E_{p}\right)$. This is also the sum of associative inhibition and associative discrimination $\left(\mathbf{I}+\mathbf{A}_{\mathbf{D}}\right)$.

both the underlying model and methods for obtaining them are different. The present difference functions do not lead directly to a computation of error rates. The two separate growth functions of $E$ are necessary for this purpose.

A more analytic description of the dynamics of similarity effects may be obtained by an inspection of the component functions of Figure 4. The function for sensory strength (V) is the same for all conditions except for minor differences introduced by differences in the criterion parameters. However, all of the three associative functions are affected by similarity. The growth of positive associative strength (A) and associative discrimination $\left(A_{D}\right)$ is clearly more rapid for the $1,300-\mathrm{Hz}$ experiment than for either of the experiments with greater similarity. The functions for $\mathrm{A}$ are quite similar in the 1,100- and $1,050-\mathrm{Hz}$ conditions. $A_{D}$ is greater for $1,100 \mathrm{~Hz}$ than for $1,050 \mathrm{~Hz}$. For associative inhibition (I), both the rate of growth and asymptotic level systematically decrease at all levels of increasing similarity. The difference in inhibition is particularly large between the 1,100 - and $1,050-\mathrm{Hz}$ experiments. Thus, the analysis indicates that the short-latency process of associative inhibition is especially vulnerable to the effect of increasing stimulus similarity. This agrees with the finding of Spiker (1978) for DRT that increasing similarity decreased both the growth rate and asymptote of functions for $I_{t}$. As indicated earlier, the inhibition interpretation of the early separation of the correct and error functions was supported by the details of the data in all three experiments.

In addition to analyzing similarity effects in terms of the theoretical concepts, it is also of interest to examine them directly in terms of response proportions. For this purpose, we use the data of the group distributions. One way of doing this is to present speed-accuracy tradeoff functions in the form of conditional error functions. In Figure 8, we present such functions of time in two ways for each experiment. In the upper panel, the functions are based on the cumulative distributions such that a point represents the conditional probability of an error given that a response has occurred by that time. Thus, the conditional error rate decreases monotonically with time in a negatively accelerated fashion, with the level depending upon similarity. In the lower panel, the functions are based only upon responses occurring within 100-msec intervals, and points represent the conditional probability of an error given that a response has occurred within the interval. The points reflect responding on the basis of new information as it becomes available. What is indicated here is that the most similar condition gains the most with time, and that similarity effects decrease with time. The function for $1,050 \mathrm{~Hz}$ is not monotonic. The inversion occurs as both the correct and error E functions are nearing asymptote. It is of little practical importance in this experiment, since the actual number of errors at long latencies is very small. The lines in Figure 8 connect points calculated from our predictions of the group distributions.

In the learning literature, it is conventional to represent the effects of stimulus similarity in the form of stimulus generalization gradients, and such
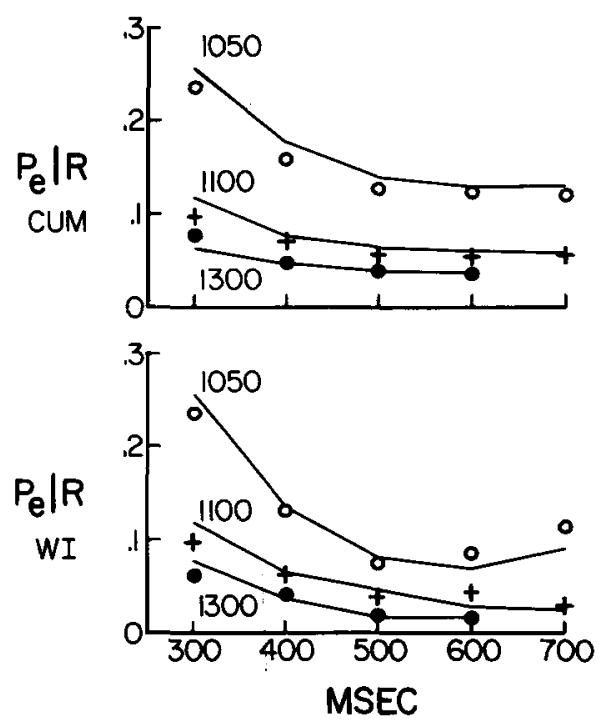

Figure 8. Speed-accuracy tradeoff functions in the form of conditional error functions. The upper figure is based on the cumulative data and gives the conditional probability of an error given that a response has occurred. The lower figure ( $P E \mid R$ within intervals) gives the conditional probability of an error given that a response has occurred within the preceding 100 -msec interval. The first points are based on all responses less than 300 msec. 


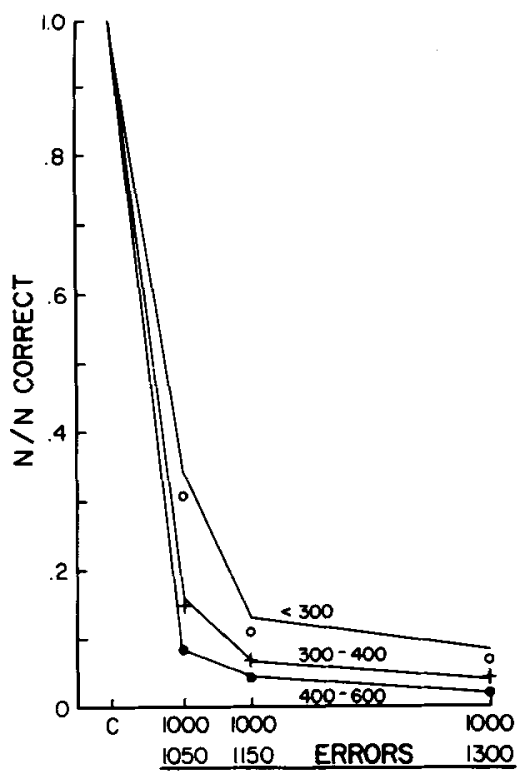

Figure 9. Relative generalization gradients over the three levels of similarity based on responses within three latency bands, $<300$ msec, 300-400 msec, and 400-600 msec. The proportions of correct responses $(C)$ are taken as unity, and errors are expressed as proportions of the numbers of correct responses. Stimulus conditions are represented on a $\log$ frequency scale.

representation is appropriate here. What is novel here is that we may present separate gradients for responses occurring with different latencies. ${ }^{4}$ In Figure 9, we present a family of such gradients. Errors are treated as generalized responses and presented as proportions of the correct responses in each experiment. Separate gradients are presented for responses before $300 \mathrm{msec}$ and for the intervals from 300 to $400 \mathrm{msec}$ and from 400 to $600 \mathrm{msec}$. Responses to the two stimuli in each condition are combined, and the scale is logarithmic with respect to the frequency of the tone paired with $1,000 \mathrm{~Hz}$. What this picture clearly shows is that the shape of the gradient depends on the latency of the response, and that its steepness increases with latency. Differences in generalization among the three similarity conditions decrease substantially with increasing latency of response. The greatest decreases in generalization are in the condition of greatest similarity. As with the conditional error functions, this also illustrates the point that the greatest gains in accuracy, achieved by delaying response, are in conditions of high similarity. Again in Figure 9, the lines connect points calculated from the predicted group distributions.

\section{Prediction of the Individual Subject Distributions}

The scaling solution, by means of which the functions for the growth of E were derived, was based on the assumption that these functions for correct responses are the same for all subjects except for individual differences in the criterion parameters. The high degree of linearity obtained in the RECs relating the subject functions to the mean scale values provides support for this assumption. However, a more complete evaluation of the applicability of the theory to the individual subjects is the prediction, from the theory, of all individual distributions. The criterion parameters, $\bar{C}$ and $\sigma$, estimated from the correct response data, also apply to the error data. However, our previous data indicate that subjects also differ in inhibitory ability. This is reflected in a single weighting factor, $\mathrm{W}_{\mathrm{I}}$, which is applied to the function for the growth of 1 . The values of $\bar{C}$ and $\sigma$ for each subject are available from the scaling analysis, but the values of $W_{I}$ must also be estimated.

The first step of the procedure is to use the criterion parameters to convert all of the theoretical functions (V, $A, A_{D}$, and $\left.I\right)$ to the scale of each subject. The criterion parameters are on the common scale, and the appropriate transformation is

$$
\mathrm{S}_{\mathrm{k}}=1 / \sigma_{\mathrm{k}}\left(\mathrm{S}_{\mathrm{c}}\right)-\overline{\mathrm{C}}_{\mathrm{k}} / \sigma_{\mathrm{k}},
$$

where $S_{c}$ is a scale value on the common scale, $\bar{C}_{k}$ and $o_{k}$ are the criterion parameters of subject $\mathrm{k}$, and $S_{k}$ is the transformed scale value on the scale of subject $k$. Actually, the whole of Equation 33 applies only to the function for $\mathrm{V}$, which is the only one in which $\bar{C}$ is specified. The remaining three functions were transformed only with respect to unit size by the slope parameter. As in the earlier analysis, the estimates of $W_{I}$ were based on only a single time for each experiment. The times, which were selected to avoid the extreme tails of error distributions, were $380 \mathrm{msec}$ for $1,100 \mathrm{~Hz}$ and $400 \mathrm{msec}$ for $1,050 \mathrm{~Hz}$. By means of the transformed functions, combined by Equation 14, excitatory strength for errors $\left(E_{e}\right)$ was computed for each subject at these times. The I components in these computations have the common weight of one. When the computed values of $E_{e}$ are compared with the obtained values of $E_{e}$ from Equation 11, the subject's weight may be inferred by assuming that any discrepancy is determined by it. Thus, for each subject, the weights were obtained by

$$
W_{I}=\frac{I-\left(E_{e o}-E_{e c}\right)}{I},
$$

where $E_{e o}$ is the obtained value of $E_{e}, E_{e c}$ is the calculated value, $I$ is the calculated value from the equal weight solution, and $\mathrm{W}_{\mathrm{I}}$ is the weight. For the $1,100-\mathrm{Hz}$ experiment, the values of $W_{1}$ varied from .43 to 1.63 , with mean of .99 and standard deviation of .28 . For the $1,050-\mathrm{Hz}$ experiment, the range was from .22 to 1.52 , with a mean of .96 and a standard deviation of .41 . Weighted functions for the growth 
of I were then obtained by multiplying each of the subjects' transformations of Equations 25 or 26 by $W_{\mathrm{I}}$. Functions for the growth of $\mathrm{E}_{\mathrm{c}}$ and $\mathrm{E}_{\mathrm{e}}$ were than obtained by Equations 12 and 15. Calculated cumulative distributions for both correct responses and errors were then obtained for each subject by Equations 6 and 7. Calculations were at $40-\mathrm{msec}$ intervals.

The calculated distributions, in general, provided very satisfactory descriptions of the subjects' empirical distributions. For correct responses in the $1,100-\mathrm{Hz}$ experiment, the proportions of variance accounted for varied from .991 to .999 . The mean proportion was .997 and the median was .998. In the $1,050-\mathrm{Hz}$ experiment, the range of proportions of variance accounted for was from .988 to .9998 . The mean was .996 and the median was .997 . For correct responses and errors combined, the proportions of variance accounted for within distributions had the same ranges. However, the means and medians were reduced by .001 . Graphs of the predicted and obtained distributions for eight representative subjects in each experiment are presented in Figure 10 for $1,100 \mathrm{~Hz}$ and in Figure 11 for $1,050 \mathrm{~Hz}$. The identifying numbers for each subject indicate the rank order of goodness of fit of the correct distribution as defined by proportion of variance accounted for. Thus, in each case, the poorest fit is in the upper left corner. For $1,100 \mathrm{~Hz}$, the third best fit is in the lower right corner, and for $1,050 \mathrm{~Hz}$, the fourth best is in that position. Subject 5 in the $1,100-\mathrm{Hz}$ experiment made no errors and none were predicted. Also, in $1,100 \mathrm{~Hz}$, subject 16 represents the poorest fit obtained to an

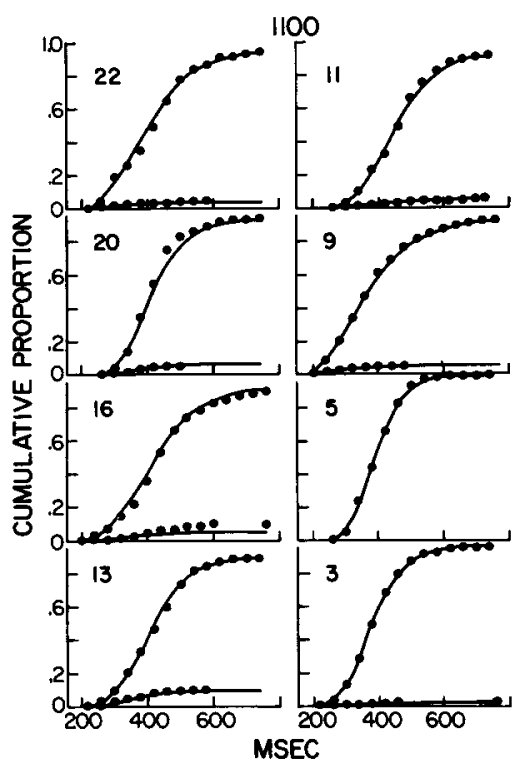

Figure 10. Predicted and obtained cumulative distributions of correct responses and errors for eight subjects in the $1,100-\mathrm{Hz}$ experiment. Upper distributions are for correct responses. error distribution. In the $1,050-\mathrm{Hz}$ experiment, subject 16 had high criterion variability and the highest error rate. Subject 13 had the lowest criterion variability and the lowest error rate in that experiment. These predicted distributions are based on three individual difference parameters estimated for each subject. $\overline{\mathrm{C}}$ and $\sigma$ were derived from the correct response data only, and $\mathrm{W}_{\mathrm{I}}$ was derived from the error data. The theory gives a substantially accurate description of individual as well as of group performance.

It is of interest to note that the above analysis assumes invariance of the theoretical functions for $V$, $A$, and $A_{D}$ over subjects. The function for $I$ is also invariant in form but subject to an individual weighting factor. While this is probably somewhat of an oversimplification, the above analysis provides quite strong support for the assumption as a close approximation of the truth. A related question concerns the extent to which the functions will remain invariant with extended practice. Of course, the present research provides no evidence concerning this question, but some evidence is available. In SRT, Grice et al. (1974) found the functions for $V$ for three stimulus intensities to be invariant across nine experimental sessions. In DRT, Grice et al. (1976) also found evidence for substantial invariance of the functions for $A$ and I with extended practice.

\section{Processing Dynamics Inferred from Individual Differences}

Since the theory provides three theoretical individual difference variables, it becomes possible to step away from the structure of the theory itself, and to

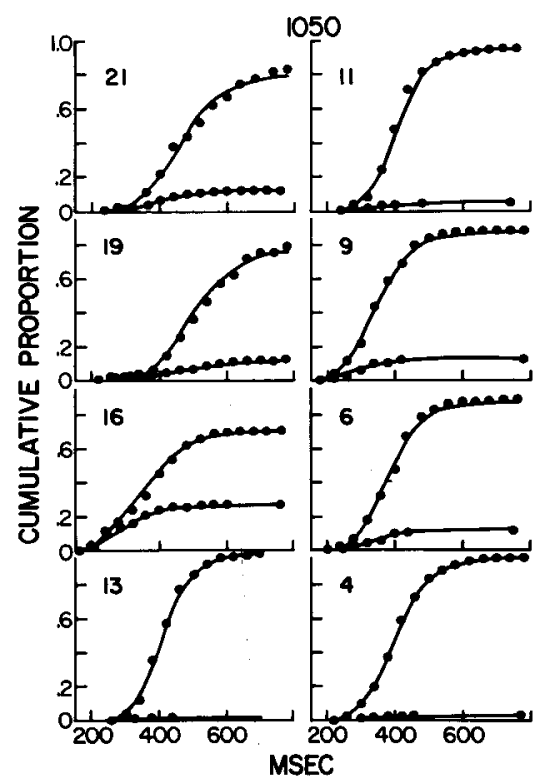

Figure 11. Predicted and obtained cumulative distributions of correct responses and errors for eight subjects in the $1,050-\mathrm{Hz}$ experiment. Upper distributions are for correct responses. 
investigate, by multiple regression analysis, the relations among these variables and also their relations with other empirical variables. To the extent that such analysis provides interesting or provocative results, the usefulness of the theory is increased. In the previous paper (Grice et al., 1977), we presented an analysis relating these variables and error rate (ER) for the 1,300-Hz deadline experiment. Such an analysis is useful only if the ER is sufficiently high to permit reasonably large individual difference variance. Another such experiment is the present $1,050-\mathrm{Hz}$ experiment. ER in the $1,300-\mathrm{Hz}$ deadline experiment was .098 , and in the $1,050-\mathrm{Hz}$ (accuracy) experiment, .122. A comparison of multiple regression analyses for these two experiments is of particular interest because the ERs were produced in different ways. In the $1,300-\mathrm{Hz}$ experiment, the discrimination was relatively easy and errors were induced by the speeding made necessary by the deadline. The $1,050-\mathrm{Hz}$ experiment was conducted under accuracy instructions, and errors resulted from the more difficult discrimination.

The intercorrelations between ER and the three theoretical variables, $\bar{C}, \sigma$, and $\mathrm{W}_{\mathrm{I}}$, are presented in Table 1. Before proceeding, we should indicate that the correlations are not artifactual in the sense that a particular pattern is assured by the estimation procedures. $\bar{C}$ and $o$ were estimated from the correct response functions only, and any degree of correlation between them would be possible. $W_{I}$ was estimated from the error functions. The negative relations with ER imply that the equal-weight solution tended to overestimate low and underestimate high ERs. However, a zero or even positive correlation would have been possible. In the $1,300-\mathrm{Hz}$ experiment, the multiple correlation (R) for predicting ER from the theoretical variables was .951 and in the $1,050-\mathrm{Hz}$ experiment, it was .967. The multiple regression equations, in standard score form, for predicting ER from the three variables are as follows:

$$
\text { for } 1,300 \mathrm{~Hz}: \hat{z}_{E R}=-.037 z_{\bar{C}}+.656 z_{o}-.552 z_{W_{I}} \text {, }
$$

for $1,050 \mathrm{~Hz}: \hat{z}_{\mathrm{ER}}=-.518 \mathrm{z}_{\overline{\mathrm{C}}}+.497 \mathrm{z}_{\mathrm{o}}-.977 \mathrm{z}_{\mathrm{W}_{\mathrm{I}}}$.

Table 1

Intercorrelations of Error Rate (ER) and the Three Theoretical Individual Difference Variables

\begin{tabular}{lccclccc}
\hline & \multicolumn{2}{c}{1,300} & Deadline Experiment & & \multicolumn{3}{c}{ 1,050 Experiment } \\
\cline { 2 - 3 } \cline { 6 - 8 } & $\overline{\mathrm{C}}$ & $\alpha$ & $\mathrm{W}_{\mathrm{I}}$ & & $\overline{\mathrm{C}}$ & $\alpha$ & $\mathrm{W}_{\mathrm{I}}$ \\
\hline $\mathrm{ER}$ & -.169 & .768 & -.651 & & .281 & .684 & -.758 \\
$\overline{\mathrm{C}}$ & & .002 & -.140 & & .208 & -.712 \\
$\alpha$ & & & -.204 & & & -.302 \\
\hline
\end{tabular}

Table 2

Values of F and Measures of Importance (U) for Regression Coefficients Predicting Error Rate from the Three Theoretical Variables

\begin{tabular}{cccccc}
\hline & \multicolumn{1}{c}{1,300 Deadline Experiment } & & \multicolumn{2}{c}{1,050 Experiment } \\
\cline { 6 - 6 } \cline { 5 - 6 } & $\mathrm{F}(1,20)$ & $\mathrm{U}(1,20)$ & & $\mathrm{F}(1,17)$ & $\mathrm{U}(1,17)$ \\
\hline$\overline{\mathrm{C}}$ & $12.52^{*}$ & .060 & & $34.64^{* *}$ & .132 \\
$\alpha$ & $86.11^{* *}$ & .412 & & $58.78^{* *}$ & .224 \\
$\mathrm{~W}_{\mathrm{I}}$ & $59.63^{* *}$ & .285 & & $116.93^{* *}$ & .446 \\
\hline
\end{tabular}

${ }^{*} p<.01 . \quad{ }^{* *} p<.001$.

A measure of the predictive importance or usefulness $(\mathrm{U})$ of a variable in a multiple regression equation is the reduction in $R^{2}$ when the equation is calculated with the variable deleted (Darlington, 1968). A related value is $F$, which tests the significance of the variable's contribution to the prediction. Values of both $U$ and $F$ for the three predictive variables in each experiment are presented in Table 2.

In the $1,300-\mathrm{Hz}$ deadline experiment, $\sigma$ has the largest weight and is most important in contributing to the regression. This confirms a major prediction of the theory. Even though estimated from correct data only, $o$ should be positively related to ER. This is readily understood with respect to the $E$ functions of Figure 3. Increases in $\sigma$ increase the size of the unit of the ordinate, resulting in a decrease in the distance between the correct and error functions in $\sigma$ units. This also increases the intersection of the error function with the criterion distribution. $W_{I}$ also contributes significantly to the regression, appropriately with a negative weight. The criterion mean $\overline{\mathrm{C}}$ contributes little but reaches significance. There was very little individual difference variance for this term in this experiment.

The pattern is quite different for the $1,050-\mathrm{Hz}$ experiment. Here all three of the variables contribute to the prediction, and the most important is $\mathrm{W}_{\mathrm{I}}$. However, $\sigma$ still contributes substantially. Even though $\mathrm{W}_{\mathrm{I}}$ is the best predictor, regression based on the two criterion parameters alone still accounts for $49 \%$ of the ER variance. The most interesting feature of this pattern is the relatively small, but significant, contribution of $\bar{C}$. The theory predicts that, other things being equal, lower criteria will lead to higher error rates. The regression coefficient for $\bar{C}$ is appropriately negative. However, the correlation coefficient between $\bar{C}$ and $E R$ is positive. In the absence of the total pattern, this could be quite puzzling. The matter may be understood by examining the partial correlations of $\overrightarrow{\mathrm{C}}$ with ER. While the simple correlation is .281 , the partial correlation with $\mathrm{W}_{\mathrm{I}}$ held constant is -.565 . When both $\mathrm{W}_{\mathrm{I}}$ and $o$ are held constant, the partial correlation is -.819 . Both negative partial correlations are significant. For $\mathrm{W}_{\mathrm{I}}$ only, $\mathrm{t}(18)=2.91, \mathrm{p}<.01$. For both $\mathrm{W}_{\mathrm{I}}$ and $\sigma$, $\mathrm{t}(17)=5.89, \mathrm{p}<.001$. These dramatic changes in 
correlation when the correlated variables are controlled represent important features of the data.

The most obvious interpretation of these individual difference patterns reveals interesting and significant features of the information processing dynamics of this situation. Possibly because of task requirements with a deadline contingency, subjects may have little freedom to adjust the criterion level, so individual differences in this variable are small and it accounts for little of the variance in error rate. Also, when the discrimination is relatively easy, inhibitory ability is of less importance in determining error rate than when the discrimination is more difficult. Associative inhibition is clearly a discriminative concept. However, it is unknown at this time the extent to which it reflects simple sensory discrimination or a more specific ability to inhibit response. We suspect that both factors are involved. In the 1,050-Hz experiment, where individual differences in inhibitory ability are of more importance, there is also more freedom to adjust the criterion level since no deadline was imposed. Thus, apparently, subjects with less inhibitory ability tend to adjust the criterion upward in compensation. While the most obvious implication of the theory is that low criteria cause errors, it is equally consistent with the theory that the criterion may be strategically increased because of a tendency to make errors for other reasons. In both situations, criterion variability was an important factor in determining error rate.

\section{DISCUSSION}

At this point, it is useful to review the current status of our efforts to apply the variable criterion principle toward the development of a general theory of response latency to simple stimuli, and to mention some questions which remain. The theory has now been extended to SRT, DRT, and CRT. All of these applications have in common the principle that the excitatory strength necessary for response evocation grows as an orderly function of time following stimulus onset until the criterion value for the trial is reached and the response is evoked. As pointed out by Grice et al. (1976), we have not so far dealt explicitly with the time of the motor component of RT, but have treated it essentially as a constant which is part of the so-called "irreducible minimum" RT. In SRT, there is a single response with a single criterion distribution. The growth of $E$ depends entirely on the growth of sensory detection (V), which is a function of stimulus intensity. In DRT (the c-reaction), there is also a single response with its criterion distribution. However, there are two stimuli-the imperative and catch stimuli. Each leads to a different function for the growth of $E$. When the pure inhib- itory strategy is used, $\mathrm{E}$ for the imperative stimulus depends entirely on V, identical to SRT. If an associative strategy is used, there is also a later associative component. The growth function for the catch stimulus also depends upon $\mathrm{V}$, but is progressively attenuated by associative inhibition, $I$. Thus, it falls progressively below that for the imperative stimulus. Errors (false alarms) occur because, on some trials, the criterion is sufficiently low that the catch stimulus function reaches criterion. The speed-accuracy tradeoff in DRT is described by the divergence of the two functions, and conditional error functions resemble those presented here for CRT.

The model for CRT represents a straightforward extension. In the two-choice situation, there are now two responses as well as two stimuli. Thus, when one stimulus is presented, the result is two functions for the growth of E-one for each response. Growth of $\mathrm{E}$ for the correct response corresponds to that for the imperative stimulus in DRT, and for the incorrect response it corresponds to that for the catch stimulus. The single additional statement that the criterion distributions of the two responses are independent generates the race model described above. The speedaccuracy tradeoff is described by the divergence of two functions as in DRT. While in all data so far observed, the correct function is continuously above the error function, errors occur because, under these circumstances, they sometimes reach criterion first. When evidence for response bias is minimal, the theoretical calculations may be collapsed over the two S-R combinations, as we have done here and in the previous paper. However, if either response or stimulus bias is present, each combination must be analyzed separately. This is also true when the stimuli differ in intensity and separate $V$ functions apply. One interesting question which arises from the presence of two simultaneously growing $E$ functions is whether, on some trials, there should not be two responses in quick succession. This does actually occur, particularly on trials ending in an error. However, such responses are not recorded with our conventional CRT procedures. It would not be correct to say that the theory really makes such a prediction. The initiation of a response changes the stimulus situation and the subject's set, and the remaining criterion value and growth function are so quickly changed that the dynamics in progress are no longer applicable. In other words, the boundary conditions of the theory do not include times following the first response.

At the level of scientific methodology, the suggestions by Link (1979) require additional comment. Use of the kind of methods he proposes would be a tidy mathematical accomplishment if it could be brought off. However, in our opinion, it would never constitute a meaningful or "cogent" test of the 
theory and the result would be scientifically trivial. In the first place, the suggestion fails to recognize the important distinction between theory construction and theory testing. We believe that explicit methods of theory construction, closely tied to data and simultaneously used as a guide for empirical research, are not only legitimate but of ten preferable to a purely deductive approach to psychological theory. Techniques which lead to identification and quantification of theoretical processes and to the discovery of the relationships between differing experimental situations are clearly directed toward the growth of a unified body of scientific knowledge. As a test of theory, Link's proposal ignores the invariances which this theory assumes and any parsimonious theory should contain. Each application of his method would generate an entirely new set of parameters. It would be extravagantly unparsimonious to do this, when an appropriately specific test should involve only a very small number. Tests of the kind we use are actually more than mere tests. This will become obvious when a test fails to confirm the existing theory. In such an instance, the tests is likely to localize the source of the failure. This will guide the theorist toward the proper revisions or extensions of the theory necessary to accommodate the new facts and lead toward the development of a more comprehensive theory. A formal model from which certain facts may be deduced and that merely succeeds or fails upon test may be mathematically sophisticated but scientifically naive.

It is Link's suggestion that our theoretical parameters be obtained from a CRT distribution. It should be emphasized that here the function parameters were not obtained from any single distribution but from many. The growth functions were based on the mean within-subjects functions for all subjects. Such data are much more stable than single distributions and result in a theory of substantially greater generality. The only parameters specific to individual distributions are the criterion parameters and $\mathrm{W}_{\mathrm{I}}$. The prior application of the scaling model is necessary for this accomplishment.

One outcome of the present investigation is that the generality of the CRT theory originally presented by Grice et al. (1977) has been increased. The types of mathematical functions which describe the components of the theory were originally defined with respect to the $1,300-\mathrm{Hz}$ deadline experiment. It was found that the same functions described the $1,300-\mathrm{Hz}$ accuracy experiment with new criterion parameters. Now it is found that the same set of functions describe additional experiments in which stimulus similarity is varied. As the nature of the theory would indicate, the result is new parameter values for the functions describing the associative concepts of the theory. Both positive associative strength and associative inhibition are influenced. However, the short- latency process of associative inhibition is particularly sensitive to increases in similarity.

Aside from the fact that component functions of the same form apply to all experiments, there are three particular matters of consistency in the data of all experiments which we find impressive. One of these is the early linearity of $E_{c}$ with a common $V$ function. This provides general support for the view that early correct responses are based on detection information only. The growth of the second process, associative strength, also was of the same form in each experiment. The second consistency had to do with the growth of $\mathrm{E}$ for errors. During the period of early linearity of the correct function with $\mathrm{V}$, the error function was not linear with $V$ and showed progressive divergence. This provides consistent support for the inhibitory interpretation of early discrimination. The third consistency also applies to errors. Once the function of I from DRT had been mapped into the CRT data, it was observed in each experiment that $E_{e}$ began gradually to exceed the value of $\mathrm{V}-\mathrm{I}$ at precisely the same time as $\mathrm{A}$ began to develop for correct responses. This consistency certainly strengthens the reasoning that there is a progressively attenuated amount of the same process present for correct responses. This is generalized associative strength.

Another matter deserves special mention. In general, when the concept of a decision criterion has been applied in discussions of the speed-accuracy tradeoff, the criterion has been treated as a single value for an experimental condition. Its level is the determiner of error rate. However, once a variable criterion is considered, attention is called to the amount of variability as a possible dynamic factor. In our original comparison of two experiments with different speed requirements, the tradeoff was found to depend primarily upon mean criterion level and slightly upon the amount of variability (Grice et al., 1977). In the present individual differences analysis, the amount of criterion variability was a substantially more important determiner of error rate than was its mean level. We suggest that any treatment of the effects of criterion level without a consideration of its variability is incomplete.

The ability of the theory to deal with individual differences is regarded as a major feature. The fact that individual performance can be described by a single model encourages belief in the validity of the individual difference dimensions. Their presence makes possible a kind of analysis of processing dynamics which would not be possible in their absence. The pattern suggests the hypothesis that criterion variability and inhibition are largely ability variables. Criterion level seems to be more under the subjects control, and to be influenced by strategy and task requirements. Possibly further applications of these variables will be found in the future. 


\section{REFERENCES}

Blough, D. S. Reaction times of pigeons on a wavelength discrimination task. Joumal of the Experimental Analysis of Behavior, 1978, 30, 163-167.

Bock, R. D., \& Jones, L. W. The measurement and prediction of judgement and choice. San Francisco: Holden-Day, 1968.

DaRLington, R. B. Multiple regression in psychology research and practice. Psychological Bulletin, 1968, 69, 161-182.

GiBson, E. J. Sensory generalization with voluntary reactions. Journal of Experimental Psychology, 1939, 24, 237-253.

Grice, G. R. A threshold model for drive. In H. H. Kendler \& J. T. Spence (Eds.), Essays in neobehaviorism: A memorial volume to Kenneth $W$. Spence. New York: Appleton-CenturyCrofts, 1971.

GRICE, G. R. Conditioning and a decision theory of response evocation. In G. H. Bower (Ed.), The psychology of learning and motivation (Vol. 5). New York: Academic Press, 1972.

GRICE, G. R. Information-processing dynamics of human eyelid conditioning. Journal of Experimental Psychology: General, 1977, 106, 71-93.

Grice, G. R., Hunt, R. R., Kushner, B. A., \& Morrow, C. Stimulus intensity, catch trial effects, and the speed-accuracy trade-off in reaction time: A variable criterion theory in interpretation. Memory \& Cognition, 1974, 2, 758-770.

Grice, G. R., Hunt, R. R., Kushner, B. A., \& Nullmeyer, R. Associative processes and strategies in disjunctive reaction time. Memory \& Cognition, 1976, 4, 433-445.

Grice, G. R., Nullmeyer, R., \& Spiker, V. A. Application of variable criterion theory to choice reaction time. Perception \& Psychophysics, 1977, 22, 431-449.

LAPPIN, J. S., \& DisCH, K. The latency operating characteristic: I. Effects of stimulus probability on choice reaction time. Journal of Experimental Psychology, 1972, 92, 419-427.

LINK, S. W. A comment on a new model for choice reaction time. Perception \& Psychophysics, 1979, 25, 443-446.

SPIKER, V. A. The effects of stimulus intensity and intensity differences on simple and disjunctive reaction time. $\mathrm{PhD}$ dissertation, University of New Mexico, 1978.

\section{NOTES}

1. In the previous paper, and in the present one as well, the criterion distributions for the two responses have equal means and variances. This fact simplifies these analyses, and the theoretical calculations are for the two stimulus-response combinations combined. However, it is by means of these parameters that the theory potentially deals with response bias. If response bias existed, their means and possibly their variances would differ.

2 . As a means of evaluating the accuracy of these computations, we have applied them to a simulated CRT experiment of 400 trials when the theoretical functions for the growth of $\mathrm{E}$ were initially known. The functions for the growth of correct and incorrect excitatory strength were arbitrary exponential growth functions whose relationship and form resembled those found in data. From the table of random normal numbers, we obtained a sample of $\mathbf{4 0 0}$ pairs of normal deviates, designating one member of each pair as the correct and the other as the incorrect criterion value for a trial. Using the two theoretical functions, the RT for each response was then calculated. Within each pair, the response with the shorter latency was the one which occurred. This gave distributions of correct responses and errors for the sample.

From the theoretical functions, predicted cumulative RT distributions for correct responses and errors were then obtained by the application of Equations 6 and 7. Excellent fits to the sample data were obtained. For the two distributions, the proportion of variance in the sample data points accounted for, within distributions, was .999.

The next step was to determine how well the theoretical functions for the growth of probability of reaching criterion could be recovered from the sample data. The cumulative theoretical probabilities were estimated from the sample by means of Equations 10 and 11. Again, excellent fits to the original theoretical values were obtained. The proportion of variance accounted for within the two functions was .998 .

The final step was to secure estimates of $\bar{C}$ and $\sigma$ from the sample data. The population values were zero for $\bar{C}$ and 1.0 for $\sigma$. The method was that described later in this article for estimating the parameters of individual subjects. Estimation was from a linear REC relating calculated correct response scale values from the sample to calculated values from the theoretical equation at corresponding time intervals. The estimate of $o$ was 1.006 , and the estimate of $\bar{C}$ was -.009 . The value of $r^{2}$ from the regression solution was .998

3. The value $1 / 2 \mathrm{~N}$ is the median of all possible values between zero and $1 / \mathrm{N}$, the lowest value available from the data. Its use to replace zero entries is a common convention in the scaling literature (Bock \& Jones, 1968). It was judged necessary here that all mean scale values for errors be based on the same subjects as the corresponding means for correct responses. For example, if the information present in the zero entries had been entirely neglected, the functions for $I$ or $A_{D}$ would have been underestimated at these points.

4. In a learning context, but employing a DRT task, Gibson (1939) found generalized responses to denied stimuli to be of shorter latency than correct responses. Since excitatory strength was supposed to decrease with generalization and latency was supposed to increase with decreased excitatory strength, this posed something of a problem for the learning theory of the time. Therefore, she suggested that long-latency false responses were "voluntarily inhibited," and that only short-latency ones "slipped through." This may be regarded as close to an early anticipation of the present theory that associative inhibition increases with time since stimulus onset.

More recently, Blough (1978), in an RT experiment with pigeons, obtained a bimodal RT distribution. He further found that responses in the early mode yielded flat generalization gradients, while those in the later mode produced typical decreasing gradients. In terms of the present theory, this suggests that the early responses were based on stimulus detection (V), while the later responses were under associative control.

(Received for publication January 17, 1978; revision accepted February 23, 1979.) 